PROCEEDINGS OF THE

AMERICAN MATHEMATICAL SOCIETY

Volume 130, Number 8, Pages 2383-2395

S 0002-9939(02)06362-1

Article electronically published on February 12, 2002

\title{
LINEAR MAPS ON OPERATOR ALGEBRAS THAT PRESERVE ELEMENTS ANNIHILATED BY A POLYNOMIAL
}

\author{
JINCHUAN HOU AND SHENGZHAO HOU
}

(Communicated by David R. Larson)

\begin{abstract}
In this paper some purely algebraic results are given concerning linear maps on algebras which preserve elements annihilated by a polynomial of degree greater than 1 and with no repeated roots and applied to linear maps on operator algebras such as standard operator algebras, von Neumann algebras and Banach algebras. Several results are obtained that characterize such linear maps in terms of homomorphisms, anti-homomorphisms, or, at least, Jordan homomorphisms.
\end{abstract}

\section{INTRODUCTION}

Let $f(x)$ be a complex polynomial of degree greater than 1 . We say that an element $a$ in an algebra is annihilated by $f(x)$ if $f(a)=0$. We say that a linear map $\phi$ from one algebra into another preserves elements annihilated by $f(x)$ if $f(\phi(a))=0$ whenever $f(a)=0$, and we say that $\phi$ preserves elements annihilated by $f(x)$ in both directions if $f(\phi(a))=0 \Leftrightarrow f(a)=0$. In [8], R. Howard gives a characterization of invertible linear maps which preserve matrices annihilated by a polynomial. For the infinite dimensional case, let $H$ be a Hilbert space and $f$ a complex polynomial of degree greater than 1. Semrl [16] proved that if $\phi$ is a unital (that is, $\phi(I)=I$ ) surjective linear map from $B(H)$ onto itself and if $\phi$ preserves operators annihilated by $f(x)$ in both directions, then $\phi$ is either an automorphism or an anti-automorphism. It is then interesting to ask if we can get similar results under the weaker assumption that $\phi$ preserves elements annihilated by a polynomial in one direction only and without the $\phi(I)=I$ assumption. It is also interesting to ask what we can say for Banach spaces and for general operator algebras. It is the aim of this paper to discuss such questions.

The questions considered here are in fact among the so-called linear preserver problems (LPP), that is, the problems of characterizing linear maps on operator algebras that leave invariant certain properties of operators such as spectrum, invertibility, solutions of an equation, subsets, relations, etc. The first papers concerning these problems discussed the spectrum-preserving linear maps on matrix algebras (i.e., the finite dimensional case) and date back to the nineteenth century [5, 10]. A great deal of effort has been devoted to the study of LPP in the last few

Received by the editors June 23, 2000 and, in revised form, March 23, 2001.

2000 Mathematics Subject Classification. Primary 47B48, 47L10, 46L05.

Key words and phrases. Operator algebras, linear preservers, homomorphisms.

The project is partially supported by NNSFC and PNSFS.

(C)2002 American Mathematical Society 
decades for operator algebras over both finite dimensional and infinite dimensional spaces (for example, see the surveys [11]-[13] as well as [1]-[4], [6]-[9], [14]-[16]). Many results have revealed the algebraic properties of linear preservers in terms of homomorphisms, anti-homomorphisms, or at least Jordan homomorphisms, and conversely, give new characterizations of homomorphisms. Recall that a linear map $\phi$ from an algebra $\mathcal{A}$ into another is called a Jordan homomorphism if $\phi\left(a^{2}\right)=\phi(a)^{2}$ for every $a \in \mathcal{A}$.

In the present paper we first consider, in Section 2, the linear maps which preserve elements annihilated by a polynomial with no repeated roots and of degree greater than 1 in the purely algebraic case. We show that if such a linear map $\phi$ is unital, then it is idempotent-preserving (Thm. 2.2). Generally, under the assumption $f(0)=0$, we get a description of the relationship between $\phi(1)$ and $\phi(a)$ for idempotents $a$ (Thm. 2.3). These allow us to give a characterization of algebraic homomorphisms in terms of preserving elements annihilated by a polynomial (Corollary 2.5). Then, in Section 3, we apply the results of Section 2 to linear maps on operator algebras. Let $\mathcal{P}_{0}$ be the set of all complex polynomials of degree greater than 1 and with no repeated roots such that $f(0)=0$. Let $\phi: \mathcal{A} \rightarrow \mathcal{B}$ be a linear map which preserves elements annihilated by a polynomial $f(x)$ in $\mathcal{P}_{0}$. We show that $\phi$ has nice algebraic properties in many situations. For example, we prove that there exist an invertible operator $T$ and a complex number $\lambda$ with $\lambda^{k}=1$ such that either $\phi(A)=\lambda T A T^{-1}$ for all $A \in \mathcal{A}$ or $\phi(A)=\lambda T A^{*} T^{-1}$ for all $A \in \mathcal{A}$ if (1) $\mathcal{A}$ and $\mathcal{B}$ are standard operator algebras acting on Banach spaces $X$ and $Y$, respectively, and $\phi$ is surjective and weakly continuous (Thm. 3.1), or, if (2) $\mathcal{A}=B(X), \mathcal{B}=B(Y)$, where $X$ has infinite multiplicity (for example, if $X=c_{0}, l_{p}, L_{p}[0,1], 1 \leq p \leq \infty$, or any infinite dimensional Hilbert space) and $\phi$ is surjective with $\phi(F) \neq 0$ for some finite rank operator $F$ (Thm. 3.6). If $\mathcal{A}$ is a von Neumann algebra and $\mathcal{B}$ is any unital Banach algebra, and if $\phi$ is bounded, then $\phi$ is in fact a Jordan homomorphism multiplied by a $k$-potent element (Thm. 3.2). Our results generalize some known results in 2, 15, 16] even for the Hilbert space case. In the case when $\mathcal{A}$ is a Banach algebra, we show that $\phi$ is a homomorphism multiplied by a $k$-potent element if and only if $\phi_{2}$ preserves elements annihilated by a polynomial in $\mathcal{P}_{0}$ (Thm. 3.8).

Note that, in our results, we do not assume that $\phi$ is unital nor that $\phi$ preserves elements annihilated by $f$ in both directions. And in many cases, we even do not assume surjectivity and any continuity.

\section{LiNEAR MAPS ON ALGEBRAS}

In this section we give some results about the characterization of linear maps between algebras which preserve the elements annihilated by a polynomial of degree greater than 1 and with no repeated roots.

If $f(x)$ is a polynomial, we let $\mathcal{Z}(f)$ denote the set of zeroes of $f$ and $G(f)$ denote the set of $\lambda \in \mathbb{C}$ such that $\lambda \mathcal{Z}(f)=\mathcal{Z}(f)$.

Theorem 2.1. Suppose $\mathcal{A}, \mathcal{B}$ are unital complex algebras and $\phi: \mathcal{A} \rightarrow \mathcal{B}$ is a unital linear map. The following are equivalent.

(1) There is a polynomial $f(x)$ with no repeated roots and $\operatorname{deg}(f) \geq 2$ such that $f(\phi(a))=0$ whenever $a \in \mathcal{A}$ and $f(a)=0$.

(2) $\phi$ is idempotent-preserving. 
(3) For every polynomial $g(x)$ with no repeated roots and $\operatorname{deg} g \geq 2, g(\phi(a))=0$ whenever $a \in \mathcal{A}$ and $g(a)=0$.

Proof. The implication (3) $\Longrightarrow(1)$ is obvious.

$(2) \Longrightarrow(3)$. Suppose $\lambda_{1}, \ldots, \lambda_{n}$ are the roots of $g$. Standard linear algebra arguments show that $g(a)=0$ if and only if there is an algebraically orthogonal family $e_{1}, \ldots, e_{n}$ of idempotents such that $1=\sum_{j=1}^{n} e_{j}$ and $a=\sum_{j=1}^{s} \lambda_{j} e_{j}$. However, if $\phi$ is idempotent-preserving, then $\phi$ also preserves orthogonality of idempotents, since idempotents $e$ and $f$ are orthogonal (i.e., $e f=f e=0$ ) if and only if $e+f$ is an idempotent.

$(1) \Longrightarrow(2)$. Suppose (1) is true. By replacing $f(x)$ with $f(\alpha+(\beta-\alpha) x)$, we may assume $f(0)=f(1)=0$. A nonzero complex number $\lambda$ such that $\lambda \mathcal{Z}(f) \subset \mathcal{Z}(f)$ must be a root of unity (since $\lambda, \lambda^{2}, \lambda^{3}, \ldots$ must be in $\mathcal{Z}(f)$ ). Let $G=\{0 \neq$ $\lambda \in \mathbb{C}: \lambda \mathcal{Z}(f) \subset \mathcal{Z}(f)\}$. It follows from the finiteness of $\mathcal{Z}(f)$ that $G$ is a finite multiplicative subgroup of the unit circle, and thus, for some positive integer $k$, $G=\left\{\lambda \in \mathbb{C}: \lambda^{k}=1\right\}=\{\lambda \in \mathbb{C}: \lambda \mathcal{Z}(f)=\mathcal{Z}(f)\}=G(f)$. It follows that $g(x)=x^{k+1}-x$ divides $f(x)$ and $G$ is the set of all nonzero roots of $g(x)$. If $e \in \mathcal{A}$ is an idempotent and $\alpha \in \mathcal{Z}(f)$, then $f(\alpha e)=f(\alpha(1-e))=0$, which implies $f(\alpha \phi(e))=f(\alpha(1-\phi(e)))=0$. Assume that $\lambda$ is a root of the minimal polynomial of $\phi(e)$. Since $\lambda \mathcal{Z}(f) \subset \mathcal{Z}(f)$ and $(1-\lambda) \mathcal{Z}(f) \subset \mathcal{Z}(f)$, we know both $\lambda$ and $1-\lambda$ are in $G$. It follows that the minimal polynomials of both $\phi(e)$ and $1-\phi(e)$ divide $g(x)$. Hence we have $g(\phi(e))=g(1-\phi(e))=0$. Suppose, for a contradiction, that the minimal polynomial of $\phi(e)$ has a root $\lambda$ with $0 \neq \lambda \neq 1$. Then $\lambda^{k}=1$ and $(1-\lambda)^{k}=1$. It follows that either $\lambda=e^{\frac{\pi}{3} i}$ or $\lambda=e^{-\frac{\pi}{3} i}$. Note that, for any $\lambda_{1}$ and $\lambda_{2}$ in $G$ and any $\alpha$ in $\mathcal{Z}(f)$, one has $f\left(\alpha\left(\lambda_{1}(1-e)+\lambda_{2} e\right)\right)=f\left(\alpha \lambda_{1}\right)(1-e)+f\left(\alpha \lambda_{2}\right) e=$ 0 . Therefore every nonzero root of the minimal polynomial of $\lambda_{1}(1-\phi(e))+\lambda_{2} \phi(e)$ is in $G$. In particular, taking $\lambda_{1}=1$ and $\lambda_{2}=\lambda^{-1}$, one gets $2-\lambda \in G$, which is a contradiction.

Remark 2.1. Theorem 2.1 cannot be generalized to the case of a polynomial with repeated roots. For example, if we let $\mathcal{A}=\left\{\left[\begin{array}{ll}\alpha & \beta \\ 0 & \alpha\end{array}\right]: \alpha, \beta \in \mathbb{C}\right\}$ and let $\phi$ : $\mathcal{A} \rightarrow M_{2}(\mathbb{C})$ be defined by $\left[\begin{array}{ll}\alpha & \beta \\ 0 & \alpha\end{array}\right] \mapsto\left[\begin{array}{cc}\alpha+\beta & 0 \\ 0 & \alpha\end{array}\right]$, then $\phi$ is obviously unital and preserves idempotents, while it does not preserve elements annihilated by $x^{2}$. If we let $\mathcal{A}=\left\{\left[\begin{array}{lll}\alpha & & 0 \\ & \beta & \\ 0 & & \gamma\end{array}\right]: \alpha, \beta, \gamma \in \mathbb{C}\right\}$ and let $\phi: \mathcal{A} \rightarrow M_{3}(\mathbb{C})$ be defined by $\left[\begin{array}{ccc}\alpha & & 0 \\ & \beta & \\ 0 & & \gamma\end{array}\right] \mapsto\left[\begin{array}{ccc}\alpha+\beta-\gamma & 0 \\ 0 & \beta & \\ 0 & & \gamma\end{array}\right]$, then $\phi$ is unital and preserves elements annihilated by $x^{2}$. But $\phi$ does not preserve idempotents.

The following lemma is technical.

Lemma 2.2. Suppose $k \geq 2$ is a positive integer, $\mathcal{B}$ is a unital algebra over $\mathbb{C}$, and $u, v \in \mathcal{B}$ are such that $v^{k+1}=v,(u-v)^{k+1}=u-v$ and

$$
\left(e^{\frac{2 \pi i s}{k}} u+\left(e^{\frac{2 \pi i t}{k}}-e^{\frac{2 \pi i s}{k}}\right) v\right)^{k+1}=e^{\frac{2 \pi i s}{k}} u+\left(e^{\frac{2 \pi i t}{k}}-e^{\frac{2 \pi i s}{k}}\right) v
$$

for $1 \leq s, t \leq k$. Then $u v=v u$, and $u=0$ implies $v=0$. 
Proof. Letting $s=t=k$, we see that $u^{k+1}=u$. Note that when $m \in \mathbb{N}$ we have $\sum_{t=1}^{k}\left(e^{\frac{2 \pi i t}{k}}\right)^{m}=\sum_{t=1}^{k}\left(e^{\frac{2 \pi i m}{k}}\right)^{t}$ equals 0 if $k$ does not divide $m$, and equals $k$ if $k$ divides $m$. It is clear that if $u=0$, we have

$$
\left(e^{\frac{2 \pi i t}{k}}-e^{\frac{2 \pi i s}{k}}\right)^{k+1} v=\left(e^{\frac{2 \pi i t}{k}}-e^{\frac{2 \pi i s}{k}}\right) v
$$

for $1 \leq s, t \leq k$. Since $k \geq 2$, this means $v=0$.

Let $w=u-v$. Since $e^{\frac{2 \pi i s}{k}} u+\left(e^{\frac{2 \pi i t}{k}}-e^{\frac{2 \pi i s}{k}}\right) v=e^{\frac{2 \pi i s}{k}} w+e^{\frac{2 \pi i t}{k}} v$, letting $s=k$, we have

$$
\left(w+e^{\frac{2 \pi i t}{k}} v\right)^{k+1}=w+e^{\frac{2 \pi i t}{k}} v
$$

for $1 \leq t \leq k$. If we multiply the left side we get $w^{k+1}$ plus terms of the form $\left(e^{\frac{2 \pi i t}{k}}\right)^{m} q_{m}$, where $q_{m}$ is the sum of all products containing $m$ factors of $v$ and $k+1-m$ factors of $w$. If we take the sum $\sum_{t=1}^{k}\left(w+e^{\frac{2 \pi i t}{k}} v\right)^{k+1}$, we see that all the terms $\left(e^{\frac{2 \pi i t}{k}}\right)^{m} q_{m}$ disappear except when $m=k$. This gives us

$$
\sum_{t=1}^{k}\left(w+e^{\frac{2 \pi i t}{k}} v\right)^{k+1}=k w^{k+1}+k q_{k}=k w+k q_{k} .
$$

On the other hand, the sum of the right hand side, i.e., $\sum_{t=1}^{k}\left(w+e^{\frac{2 \pi i t}{k}} v\right)$, is equal to $k w$. It follows that $q_{k}=0$. However, a simple computation (using $v^{k+1}=v$ ) shows that

$$
0=v q_{k}-q_{k} v=v w-w v .
$$

Hence we have $v w=w v$, from which it follows that $u v=v u$.

Recall that $\mathcal{P}_{0}$ is the set of all polynomials $f$ with no repeated roots and of degree greater than 1 such that $f(0)=0$.

Theorem 2.3. Suppose $\mathcal{A}, \mathcal{B}$ are unital complex algebras and $\phi: \mathcal{A} \rightarrow \mathcal{B}$ is a linear map, and suppose $f(x)$ is a polynomial in $\mathcal{P}_{0}$ such that $f(\phi(a))=0$ whenever $a \in \mathcal{A}$ and $f(a)=0$. Then there exist a positive integer $k$, a polynomial $h(x)$ and an orthogonal family $p_{1}, \ldots, p_{k}$ of idempotent in $\mathcal{B}$ such that

(1) $f(x)=x h\left(x^{k}\right)$;

(2) if $\lambda$ is any root of $x^{k}-1$, then $\lambda \mathcal{Z}(f)=\mathcal{Z}(f)$;

(3) $g(\phi(a))=0$ for every idempotent $a \in \mathcal{A}$, where $g(x)=x^{k+1}-x$;

(4) $\phi(1)=\sum_{s=1}^{k} e^{\frac{2 \pi s i}{k}} p_{s}$

(5) $p_{s}$ commutes with $\phi(a)$ for $1 \leq s \leq k$ and for every idempotent $a \in \mathcal{A}$;

(6) $\phi(a)=\sum_{s=1}^{k} \phi(a) p_{s}$ for every idempotent $a \in \mathcal{A}$.

Proof. Let $G=G(f)=\{0 \neq \lambda: \lambda \mathcal{Z}(f)=\mathcal{Z}(f)\} . \quad G$ is a finite multiplicative subgroup of the unit circle, and thus, for some positive integer $k, G=\{\lambda \in \mathbb{C}$ : $\left.\lambda^{k}=1\right\}$. It follows that (1) and (2) must be true. As in the proof of Theorem 2.1, we see that if $a$ is an idempotent in $\mathcal{A}$, and $\lambda \neq 0$ is a root of the minimal polynomial of $\phi(a)$, then $\lambda \in G$. Hence (3) is true. Since $g(\phi(1))=0$, (4) must be true, with possibly $p_{s}=0$ for some values of $s$.

If $k=1$, it follows from (3) that $\phi$ is idempotent-preserving, and, thus, for every idempotent $a \in \mathcal{A}, \phi(a)$ and $\phi(1-a)$ must be orthogonal idempotents whose sum is 
$\phi(1)$. This implies that, for every idempotent $a \in \mathcal{A}, \phi(a) \phi(1)=\phi(1) \phi(a)=\phi(a)$, which yields both (5) and (6).

On the other hand, suppose $k \geq 2$. It follows, for every idempotent $a \in \mathcal{A}$, for every $\alpha \in \mathcal{Z}(f)$ and all integers $1 \leq s, t \leq k$, that $f\left(\alpha\left[e^{\frac{2 \pi i s}{k}} 1+\left(e^{\frac{2 \pi i t}{k}}-e^{\frac{2 \pi i s}{k}}\right) a\right]\right)=$ 0 , so any nonzero root of the minimal polynomial of $e^{\frac{2 \pi i s}{k}} \phi(1)+\left(e^{\frac{2 \pi i t}{k}}-e^{\frac{2 \pi i s}{k}}\right) \phi(a)$ must be in $G$. Hence the hypothesis of Lemma 2.2 holds, with $u=\phi(1)$ and $v=\phi(a)$. Thus (5) and (6) follow from Lemma 2.2.

For an algebra $\mathcal{A}$ over $\mathbb{C}$ we denote by $\mathcal{A} \otimes M_{n}(\mathbb{C})$ the algebra of $n \times n$ matrices with entries in $\mathcal{A}$. If $\phi: \mathcal{A} \rightarrow \mathcal{B}$ is a linear map, we denote by $\phi_{n}$ the linear map from $\mathcal{A} \otimes M_{n}(\mathbb{C})$ into $\mathcal{B} \otimes M_{n}(\mathbb{C})$ which maps every matrix $\left(a_{i j}\right)_{n \times n}$ to $\left(\phi\left(a_{i j}\right)\right)_{n \times n}$. By use of Theorems 2.1 and 2.3 we can get a characterization of homomorphisms between algebras.

Corollary 2.4. Let $\mathcal{A}$ and $\mathcal{B}$ be unital complex algebras. Let $\phi: \mathcal{A} \rightarrow \mathcal{B}$ be a linear map. If $\phi_{2}$ preserves the elements annihilated by a polynomial $f(x) \in \mathcal{P}_{0}$, then there is a Jordan homomorphism $\psi: \mathcal{A} \rightarrow \mathcal{B}$ such that $\psi(c) \psi\left(c^{-1} a c\right)=\psi(a) \psi(c)$ holds for any $a$ and invertible element $c$ in $\mathcal{A}$, and such that $\phi=\phi(1) \psi=\psi \phi(1)$.

Proof. Obviously, $\phi$ preserves elements annihilated by $f$, too. Take the orthogonal family of idempotents $p_{1}, \ldots, p_{k}$ in $\mathcal{B}$ as that in Theorem 2.3. Then $\phi(1)=$ $\sum_{s=1}^{k} e^{\frac{2 \pi s i}{k}} p_{s}, p=\sum_{s=1}^{k} p_{s}=\phi(1)^{k}$, and for any idempotent $e$ in $\mathcal{A}$ we have $\phi(1) \phi(e)=\phi(e) \phi(1)$ and $\phi(e)=p \phi(e)=\phi(e) p$. We claim that these hold true for all elements of $\mathcal{A}$. In fact, for any $a \in \mathcal{A}$, if we take an idempotent $A=\left[\begin{array}{ll}1 & a \\ 0 & 0\end{array}\right]$ and write $\phi_{2}(1)=\left[\begin{array}{cc}\phi(1) & 0 \\ 0 & \phi(1)\end{array}\right]$, we get

$$
\left[\begin{array}{cc}
\phi(1)^{2} & \phi(1) \phi(a) \\
0 & 0
\end{array}\right]=\phi_{2}(1) \phi_{2}(A)=\phi_{2}(A) \phi_{2}(1)=\left[\begin{array}{cc}
\phi(1)^{2} & \phi(a) \phi(1) \\
0 & 0
\end{array}\right]
$$

and

$$
\left[\begin{array}{cc}
\phi(1) & p \phi(a) \\
0 & 0
\end{array}\right]=\phi_{2}(A)^{k+1}=\phi_{2}(A)=\left[\begin{array}{cc}
\phi(1) & \phi(a) \\
0 & 0
\end{array}\right],
$$

from which it follows that $\phi(1) \phi(a)=\phi(a) \phi(1)$ and $\phi(a)=p \phi(a)$. If one takes $A=$ $\left[\begin{array}{ll}1 & 0 \\ a & 0\end{array}\right]$, one get $\phi(a)=\phi(a) p$. Hence the range of $\phi$ is contained in $\mathcal{B}_{1}=p \mathcal{B} p$, which is a subalgebra of $\mathcal{B}$ with unit $p$. Moreover, every $p_{s}$ commutes with $\phi(a)$ for all $a \in \mathcal{A}$. Let $b_{0}=\sum_{s=1}^{k} e^{-\frac{2 \pi s i}{k}} p_{s}$ and $\psi=b_{0} \phi$. Then $\phi=\phi(1) \psi$. Regard $\phi$ as a linear map from $\mathcal{A}$ into $\mathcal{B}_{1}$. It follows from Theorem 2.3 (2) that both $\psi$ and $\psi_{2}$ are unital and still preserve elements annihilated by $f$, since $e^{-\frac{2 \pi s i}{k}} \mathcal{Z}(f)=\mathcal{Z}(f)$. So, by Theorem 2.1, both $\psi$ and $\psi_{2}$ are idempotent-preserving. We prove that $\psi$ is indeed a Jordan homomorphism.

For any element $a$ and invertible element $c$ in $\mathcal{A},\left[\begin{array}{cc}a & c \\ c^{-1}\left(a-a^{2}\right) & 1-c^{-1} a c\end{array}\right]$ is an idempotent in $\mathcal{A} \otimes M_{2}$. So, $\left[\begin{array}{cc}\psi(a) & \psi(c) \\ \psi\left(c^{-1}\left(a-a^{2}\right)\right) & 1-\psi\left(c^{-1} a c\right)\end{array}\right]$ is an idempotent. Hence we have

$$
\psi(a)^{2}+\psi(c) \psi\left(c^{-1}\left(a-a^{2}\right)\right)=\psi(a)
$$


and

$$
\psi(a) \psi(c)+\psi(c)\left(1-\psi\left(c^{-1} a c\right)\right)=\psi(c) .
$$

Let $c=1$ in (2.1). We get

$$
\psi\left(a^{2}\right)=\psi(a)^{2},
$$

that is, $\psi$ is a Jordan homomorphism. It follows from (2.2) that

$$
\psi(c) \psi\left(c^{-1} a c\right)=\psi(a) \psi(c),
$$

completing the proof.

Corollary 2.5. Let $\mathcal{A}$ and $\mathcal{B}$ be unital complex algebras. Let $\phi: \mathcal{A} \rightarrow \mathcal{B}$ be a linear map and $f(x)$ a polynomial in $\mathcal{P}_{0}$. The following statements are equivalent.

(1) $\phi_{3}$ preserves elements annihilated by $f(x)$.

(2) There exist a homomorphism $\psi: \mathcal{A} \rightarrow \mathcal{B}$ and an n-potent element $b$ for some integer $n \geq 2$ such that the nonzero roots of a minimal polynomial of $b$ are in $G(f)$ and $\phi=b \psi=\psi b$.

Proof. (2) $\Longrightarrow(1)$. Write $\phi=b \psi$, where $\psi$ is homomorphism and $b$ is an $n$-potent, i.e., $b^{n}=b$. Let $\left\{\lambda_{1}, \ldots, \lambda_{l}\right\}$ be the set of nonzero roots of the minimal polynomial of $b$. Then there exists a set of mutually orthogonal idempotents $\left\{e_{1}, \ldots, e_{l}\right\}$ such that $b=\sum_{s=1}^{l} \lambda_{s} e_{s}$ and $e_{s} \psi(a)=\psi(a) e_{s}$ for all $a \in \mathcal{A}$. If $a \in \mathcal{A}$ and $f(a)=0$, then $f(\phi(a))=f\left(\sum_{s=1}^{l} \lambda_{s} e_{s} \psi(a)\right)=\sum_{s=1}^{l} \psi\left(f\left(\lambda_{s} a\right)\right) e_{s}=0$, since $\psi$ is homomorphic and $f\left(\lambda_{s} a\right)=0$, by the assumption that $\lambda_{s} \mathcal{Z}(f)=\mathcal{Z}(f)$ for $1 \leq s \leq l$. As $\psi_{3}$ is still a homomorphism, one easily checks that $\phi_{3}$ preserves elements annihilated by $f$, too.

$(1) \Longrightarrow(2)$. Since $\phi_{2}$ also preserves the elements annihilated by $f$, by Corollary 2.4 , there exist a $(k+1)$-potent element $b$ and a Jordan homomorphism $\psi$ satisfying equation (2.3) such that $\phi=b \psi=\psi b$, where $k$ is the degree of the group $G(f)$. We prove that $\psi$ is indeed a homomorphism.

Without loss of generality we may assume $\psi$ is unital (otherwise, replace $\mathcal{B}$ with $\mathcal{B}_{1}=p \mathcal{B} p$ as in the proof of Corollary 2.4). Then $\psi_{3}$ is idempotent-preserving. For any elements $a, w$ and invertible $c$ in $\mathcal{A}$,

$$
\left[\begin{array}{ccc}
\psi(a) & \psi(c) & 0 \\
\psi\left(c^{-1}\left(a-a^{2}\right)\right) & 1-\psi\left(c^{-1} a c\right) & 0 \\
\psi(w a-w) & \psi(w c) & 1
\end{array}\right]
$$

is idempotent since

$$
\left[\begin{array}{ccc}
a & c & 0 \\
c^{-1}\left(a-a^{2}\right) & 1-c^{-1} a c & 0 \\
w a-w & w c & 1
\end{array}\right]
$$

is. Thus we have

$$
\psi(w a-w) \psi(c)+\psi(\omega c)\left(1-\psi\left(c^{-1} a c\right)\right)+\psi(w c)=\psi(w c),
$$

that is,

$$
\psi(w a) \psi(c)-\psi(w) \psi(c)+\psi(w c)-\psi(w c) \psi\left(c^{-1} a c\right)=0 .
$$


It is well known that a unital Jordan homomorphism $\psi$ is invertibility-preserving and $\psi\left(c^{-1}\right)=\psi(c)^{-1}$. So, by (2.3) we have $\psi\left(c^{-1} a c\right)=\psi(c)^{-1} \psi(a) \psi(c)$, and (2.4) becomes

$$
\psi(w a) \psi(c)-\psi(w) \psi(c)+\psi(w c)-\psi(w c) \psi(c)^{-1} \psi(a) \psi(c)=0 .
$$

Taking $w=c^{-1}$ in (2.5), we see that

$$
\psi\left(c^{-1} a\right)=\psi(c)^{-1} \psi(a)
$$

for all $a$ and invertible $c$. Using (2.5) again, we get $\psi(w a)=\psi(w) \psi(a)$, that is, $\psi$ is a homomorphism.

\section{LiNEAR MAPS ON OPERATOR ALGEBRAS}

In this section, we apply the results in Section 2 to the linear maps from a unital operator algebra into another which preserve elements annihilated by a polynomial in $\mathcal{P}_{0}$, that is, a polynomial of degree greater than 1 and with no repeated roots such that $f(0)=0$. The question we consider here is mainly to determine when the structure of such linear preservers can be described by homomorphisms, antihomomorphisms, or, at least, Jordan homomorphisms.

The following theorem gives a characterization of all weakly continuous surjective linear maps on standard operator algebras which preserve elements annihilated by a polynomial in $\mathcal{P}_{0}$. Recall that a closed subalgebra $\mathcal{A}$ in $B(X)$ is called a standard operator algebra if it contains the identity $I$ and the set $\mathcal{F}(X)$ of all finite rank operators. Of course $B(X)$ itself is a standard operator algebra. We say that a linear map $\phi: \mathcal{A} \rightarrow \mathcal{B}$ commutes with a polynomial $f(x)$ if $f(\phi(a))=\phi(f(a))$ for all elements $a \in \mathcal{A}$.

Theorem 3.1. Let $\mathcal{A}$ and $\mathcal{B}$ be standard operator algebras on Banach spaces $X$ and $Y$, respectively. Suppose $\phi: \mathcal{A} \rightarrow \mathcal{B}$ is a linear surjective map which is continuous in the weak operator topology and $f(x)$ is a polynomial with no repeated roots and $\operatorname{deg} f \geq 2$ such that $f(0)=0$. Then the following are equivalent.

(1) $\phi$ commutes with $f(x)$.

(2) $\phi$ preserves elements annihilated by $f(x)$.

(3) There exists a scalar $\lambda$ which is a root of 1 satisfying $\lambda \mathcal{Z}(f)=\mathcal{Z}(f)$, and either there exists an invertible operator $T \in B(X, Y)$ such that $\phi(A)=\lambda T A T^{-1}$ for all $A \in B(X)$, or there exists an invertible operator $T \in B\left(X^{*}, Y\right)$ such that $\phi(A)=\lambda T A^{*} T^{-1}$ for all $A \in B(X)$. In the last case both $X$ and $Y$ are reflexive.

Proof. (1) $\Longrightarrow(2)$. Obvious.

$(3) \Longrightarrow(1)$. Suppose (3). Let $k$ be the degree of the group $G(f)$ and let $\operatorname{deg} f=n$. The condition $\lambda \mathcal{Z}(f)=\mathcal{Z}(f)$ implies that $k$ divides $n-1$, since $f(0)=0$. So $f(\lambda x)=\lambda f(x)$. If $\phi(A)=\lambda T A T^{-1}$, then $f(\phi(A))=T f(\lambda A) T^{-1}=\phi(f(A))$, that is, (1) is true.

$(2) \Longrightarrow(3)$. Suppose that $\phi: \mathcal{A} \rightarrow \mathcal{B}$ is a weakly continuous surjective linear map which preserves elements annihilated by the polynomial $f(x)$. We claim that $\phi(I)=\lambda I$ for some scalar $\lambda$ satisfying $\lambda \mathcal{Z}(f)=\mathcal{Z}(f)$ and $\lambda^{k}=1$ for some $k \geq 1$. Take $k$ the same as in Theorem 2.3. Then, for every idempotent $A \in \mathcal{A}$ one has $\phi(A)^{k}=\phi(A)$ and $\phi(I) \phi(A)=\phi(A) \phi(I)$. Notice that every rank one operator can be represented as a linear combination of at most two rank one idempotent 
operators. The weak continuity of $\phi$ and the weak density of all finite rank operators in $B(X)$ together imply that

$$
\phi(I) \phi(S)=\phi(S) \phi(I)
$$

for every $S \in \mathcal{A}$. Thus $\phi(I)$ is the identity multiplied by a scalar, since $\mathcal{F}(X) \subset$ $\mathcal{A}$. It is clear that $\phi(I) \neq 0$, for otherwise Theorem 2.3 (6) would imply that $\phi(A)=\phi(A) \phi(I)=0$ for all idempotents $A$, and consequently $\phi=0$. Therefore we must have $\phi(I)=\lambda I$ for some $\lambda \neq 0$ with $\lambda^{k}=1$. By Theorem 2.3 (2) and (4), $\lambda \mathcal{Z}(f)=\mathcal{Z}(f)$.

Let $\psi=\lambda^{-1} \phi$. Then $\psi: \mathcal{A} \rightarrow \mathcal{B}$ is a weakly continuous surjective unital linear map which also preserves elements annihilated by $f(x)$, as $\lambda^{-1} \mathcal{Z}(f)=\mathcal{Z}(f)$. By Theorem 2.1, $\psi$ is idempotent-preserving. It follows from [3, Theorem 3.3] that $\psi$ is either an isomorphism or an anti-isomorphism. Therefore, either there is an invertible operator $T \in B(X, Y)$ such that

$$
\phi(A)=\lambda T A T^{-1}
$$

for every $A \in \mathcal{A}$, or there is an invertible operator $T \in B\left(X^{*}, Y\right)$ such that

$$
\phi(A)=\lambda T A^{*} T^{-1}
$$

for every $A \in \mathcal{A}$, with $\lambda^{k}=1$. It is clear that in the last case, $X$ and $Y$ are reflexive (for the proof of reflexivity, see 3, Prop. 3.1] or [7, Thm. 1.4]). The proof is finished.

Remark 3.1. Theorem 3.1 remains true if the assumption of weak continuity on the linear map $\phi$ is replaced by the assumption that $\phi$ is continuous in the strong operator topology or in the $\sigma$-weak operator topology.

Next we consider linear maps on von Neumann algebras.

Theorem 3.2. Let $\mathcal{A}$ be a von Neumann algebra and $\mathcal{B}$ a unital Banach algebra, and let $\phi: \mathcal{A} \rightarrow \mathcal{B}$ be a bounded linear map. Let $f(x)$ be a polynomial with no repeated roots and $\operatorname{deg} f \geq 2$ such that $f(0)=0$. Then the following statements are equivalent.

(1) $\phi$ commutes with $f(x)$.

(2) $\phi$ preserves elements annihilated by $f(x)$.

(3) There exist an $r$-potent element $B \in \mathcal{B}$ with spectrum contained in $\{\lambda$ : $\lambda \mathcal{Z}(f)=\mathcal{Z}(f)\}$ and a Jordan homomorphism $\psi$ from $\mathcal{A}$ into $\mathcal{B}$ such that $\phi=$ $B \psi=\psi B$.

Proof. It is obvious that $(1) \Longrightarrow(2)$.

$(3) \Longrightarrow(1)$. Say the spectrum $\operatorname{Sp}(B)=\left\{\lambda_{1}, \ldots, \lambda_{l}\right\}$. There is an orthogonal family $P_{1}, \ldots, P_{l}$ of idempotents in $\mathcal{B}$ such that $B=\sum_{s=1}^{l} \lambda_{s} P_{s}$. As every $\lambda_{s}$ satisfies $\lambda_{s} \mathcal{Z}(f)=\mathcal{Z}(f)$, one has $B^{k+1}=B$, where $k \geq 1$ is the integer as in Theorem 2.3. It is clear that $P_{s} \psi(A)=\psi(A) P_{s}$. Now if $A$ is any element in $\mathcal{A}$, then, since $\psi$ is a Jordan homomorphism, one has $f(\phi(A))=\sum_{s=1}^{l} f\left(\lambda_{s} \psi(A) P_{s}\right)=$ $\sum_{s=1}^{l} \lambda_{s} f(\psi(A)) P_{s}=\sum_{s=1}^{l} \lambda_{s} P_{s} \psi(f(A))=\phi(f(A))$.

$(2) \Longrightarrow(3)$. Denote the unit by $I$ for both $\mathcal{A}$ and $\mathcal{B}$. By Theorem 2.3, there is an orthogonal family $P_{1}, \ldots, P_{k-1}$ of idempotents in $\mathcal{B}$ commuting with every $\phi(A)$, and $\phi(A)=\phi(A) \sum_{s=1}^{k-1} P_{s}$ whenever $A^{2}=A$, while $\phi(I)=\sum_{s=1}^{k-1} e^{\frac{2 \pi s i}{k-1}} P_{s}$. Let $P=\sum_{s=1}^{k-1} P_{s}$ and $B_{0}=\phi(I)+(I-P)$. Then $P^{2}=P, B_{0}^{k-1}=I$, and $B_{0} \phi(A)=\phi(A) B_{0}=\phi(I) \phi(A)$ if $A^{2}=A$. It is well known that the subspace 
$\mathcal{S}$ of all linear combinations of projections (i.e., self-adjoint idempotents) in a von Neumann algebra is a dense subset under the norm topology. Since $\phi$ is continuous, we get $\phi(S)=\phi(S) P=P \phi(S)$ and $B_{0} \phi(S)=\phi(S) B_{0}=\phi(I) \phi(S)$ for all $S$ in $\mathcal{A}$. Let $\mathcal{B}_{1}=P \mathcal{B P}$ and $\psi=B_{0}^{-1} \phi$. It is clear that $\psi: \mathcal{A} \rightarrow \mathcal{B}$ is bounded with range contained $\mathcal{B}_{1}$, and preserves element annihilated by $f$, since $\mathcal{B}_{1}$ is a Banach algebra with unit $P$ and $\psi(I)=P$. By Theorem $2.1 \psi$ is idempotent-preserving and hence transforms a set of orthogonal idempotents of $\mathcal{A}$ into a set of orthogonal idempotents of $\mathcal{B}_{1} \subset \mathcal{B}$.

Now, if $A=A^{*}$ is a self-adjoint element in the von Neumann algebra $\mathcal{A}$, then $A$ is the limit of a sequence of linear combinations of orthogonal projections and consequently, by continuity of $\psi, \psi(A)$ is the limit of a sequence of linear combinations of orthogonal idempotents. It turns out that $\psi\left(A^{2}\right)=\psi(A)^{2}$ holds for self-adjoint elements $A$. Replacing $A$ by $C+D$ in this relation, where $C$ and $D$ are self-adjoint, we get $\psi(C D+D C)=\psi(C) \psi(D)+\psi(D) \psi(C)$. For any $S \in \mathcal{A}$, let $C=\frac{1}{2}\left(S+S^{*}\right)$ and $D=\frac{1}{2 i}\left(S-S^{*}\right)$; then $C, D$ are self-adjoint and $S=C+i D$. Hence

$$
\begin{aligned}
\psi\left(S^{2}\right) & =\psi\left(C^{2}-D^{2}+i(C D+D C)\right) \\
& =\psi(C)^{2}-\psi(D)^{2}+i(\psi(C) \psi(D)+\psi(D) \psi(C))=\psi(S)^{2},
\end{aligned}
$$

that is, $\psi$ is a Jordan homomorphism and $\phi=B_{0} \psi=B \psi$ with $B=\phi(I)$.

Corollary 3.3. Let $\mathcal{A}, \mathcal{B}, \phi$ and $f$ be the same as in Theorem 3.2. If $\phi$ is surjective, then the following statements are equivalent.

(1) $\phi$ commutes with $f(x)$.

(2) $\phi$ preserves elements annihilated by $f(x)$.

(3) There exists an invertible r-potent element $B$ in the center of $\mathcal{B}$ satisfying $\operatorname{Sp}(B) \subset(\lambda: \lambda \mathcal{Z}(f)=\mathcal{Z}(f)\}$, and there exists a Jordan homomorphism $\psi$ from $\mathcal{A}$ onto $\mathcal{B}$ such that $\phi=B \psi=\psi B$. Here the identity element is denoted again by $I$ for both $\mathcal{A}$ and $\mathcal{B}$.

Proof. Let $B=\phi(I)$ and $P=\sum_{s=1}^{k-1} P_{s}$ as in the proof of Theorem 3.2. Since $\phi$ is surjective, $B$ and $P$ commute with every element in $\mathcal{B}$, i.e., both $B$ and $P$ are in the center of $\mathcal{B}$. Moreover, $\phi(S)=\phi(S) P$ holds for every element $S$ in $\mathcal{A}$. This implies that $P=I$ and hence $\phi(I)$ is invertible. Now let $\psi=B^{-1} \phi$ and apply Theorem 3.2 .

Notice that, in the above corollary, if $\mathcal{B}$ is a factor, then $B=\lambda I$ for some scalar $\lambda$ with $\lambda^{k-1}=1$. If $\mathcal{B}$ is prime, then the Jordan homomorphism onto $\mathcal{B}$ is either a homomorphism or an anti-homomorphism. So if $\mathcal{B}$ is a factor and prime, then $\phi$ is a homomorphism or anti-homomorphism multiplied by a scalar which is a root of 1. In particular, we have

Corollary 3.4. Let $H$ and $K$ be Hilbert spaces and $\phi: B(H) \rightarrow B(K)$ a bounded surjective linear map. Let $f(x)$ be a polynomial of degree greater than 1 and with no repeated roots such that $f(0)=0$. Then the following are equivalent.

(1) $\phi$ preserves elements annihilated by $f(x)$.

(2) There exist a scalar $\lambda$ which is a root of 1 satisfying $\lambda \mathcal{Z}(f)=\mathcal{Z}(f)$ and an invertible operator $T \in B(H, K)$ such that either $\phi(A)=\lambda T A T^{-1}$ for every $A$, or $\phi(A)=\lambda T A^{t} T^{-1}$ for every $A$, where $A^{t}$ denotes the transpose of $A$ relative to a basis. 
Comparing Theorem 3.1 with Corollary 3.3, we weaken the assumption of $\phi$ being weakly continuous to that of $\phi$ being bounded for the case $\mathcal{A}=B(H)$ and $\mathcal{B}=B(K)$ with $H$ and $K$ being Hilbert spaces. After the next lemma, we can get a stronger one by omitting the assumption of continuity.

We say that a Banach space $X$ has infinite multiplicity if $X$ is isomorphic to $X \oplus X$ and to an infinite direct sum $X^{(\infty)}$ of copies of $X$ in such a way that arbitrary permutations of coordinates and transformations of the form $A^{(\infty)}$ (with $A \in B(X)$ ) are bounded operators, and such that, for any bounded operators $A$ and $B$ on $X, A^{(\infty)} \oplus B^{(\infty)}$ acting on $X^{(\infty)} \oplus X^{(\infty)}$ is similar to $(A \oplus B)^{(\infty)}$ acting on $(X \oplus X)^{(\infty)}$. Infinite dimensional $c_{0}$-space, infinite dimensional $l^{p}$-space, $L^{p}[0,1]$, $1 \leq p \leq \infty$, and infinite dimensional Hilbert spaces are examples of Banach spaces of infinite multiplicity [6].

Lemma 3.5. Let $X$ and $Y$ be complex Banach spaces and $\phi: B(X) \rightarrow B(Y)$ a linear map. Let $f(x)$ be a polynomial of degree greater than 1 and with no repeated roots such that $f(0)=0$. If $\phi$ preserves elements annihilated by $f$ and if $X$ has infinite multiplicity, then $\phi(A) \phi(I)=\phi(I) \phi(A)$ for every $A$ in $B(X)$, and there exists an idempotent-preserving linear map $\psi: B(X) \rightarrow B(Y)$ such that $\phi=\phi(I) \psi$.

Proof. It follows from Theorem 2.3 that there is an orthogonal family $P_{1}, \ldots, P_{k-1}$ of idempotent operators in $B(Y)$ such that $P_{s}$ commutes with every $\phi(A)$ and $\phi(A)=\phi(A) \sum_{s=1}^{k-1} P_{s}$ whenever $A^{2}=A$, and $\phi(I)=\sum_{s=1}^{k-1} e^{\frac{2 \pi s i}{k-1}} P_{s}$. Let $B=\phi(I)$ and $P=\sum_{s=1}^{k-1} P_{s}$. Then $B^{k}=B$ and $P^{2}=P$. Since $X$ has infinite multiplicity, by [6], every $S \in B(X)$ is a difference of sums of idempotents in $B(X)$. This implies that $\phi(S) B=B \phi(S)$ and $\phi(S)=\phi(S) P=P \phi(S)$ hold for all $S \in B(X)$. Let $B_{0}=B+(I-P)$ and $\psi=B_{0}^{-1} \phi$. Then $\psi$ preserves elements annihilated by $f$, and $\psi(I)=P$. If we let $Y_{0}=\operatorname{ran} P$, the range of $P$, and $\theta: B(X) \rightarrow B\left(Y_{0}\right)$ defined by $\theta(S)=\left.\psi(S)\right|_{Y_{0}}$, then $\theta$ is unital, and therefore $\theta$, as well as $\psi$, is idempotentpreserving, by Theorem 2.1. It is clear that $\phi=B \psi$.

Theorem 3.6. Let $X, Y, \phi$ and $f$ be as in Lemma 3.5. If $\phi$ is surjective and $\left.\phi\right|_{\mathcal{F}(X)} \neq 0$, then $\phi$ preserves elements annihilated by $f(x)$ if and only if there is a scalar $\lambda$ which is a root of 1 satisfying $\lambda \mathcal{Z}(f)=\mathcal{Z}(f)$ and either there is an invertible operator $T \in B(X, Y)$ such that $\phi(A)=\lambda T A T^{-1}$ for all $A \in B(X)$ or there exists an invertible operator $T \in B\left(X^{*}, Y\right)$ such that $\phi(A)=\lambda T A^{*} T^{-1}$ for all $A \in B(X)$. In the last case both $X$ and $Y$ are reflexive.

Proof. By Lemma 3.5 it is obvious that $\phi(I)=\lambda I$ for some complex number $\lambda$ with $\lambda^{k}=1$ and $\lambda \mathcal{Z}(f)=\mathcal{Z}(f)$ such that $\phi=\lambda \psi$, where $\psi$ is surjective and idempotent-preserving. We have to prove that $\psi$ is isomorphic or anti-isomorphic.

If $A \in B(X)$ is an idempotent of rank one, we first show that $\psi(A)$ also has rank at most one. Let $\mathcal{M}_{1}=A B(X) A, \mathcal{M}_{2}=A B(X)(I-A), \mathcal{M}_{3}=(I-A) B(X) A$ and $\mathcal{M}_{4}=(I-A) B(X)(I-A)$. Thus $B(X)=\mathcal{M}_{1} \oplus \mathcal{M}_{2} \oplus \mathcal{M}_{3} \oplus \mathcal{M}_{4}$. Similarly, $B(Y)=\mathcal{N}_{1} \oplus \mathcal{N}_{2} \oplus \mathcal{N}_{3} \oplus \mathcal{N}_{4}$ where $\mathcal{N}_{1}=\psi(A) B(Y) \psi(A), \mathcal{N}_{2}=\psi(A) B(Y)(I-\psi(A))$, $\mathcal{N}_{3}=(I-\psi(A)) B(Y) \psi(A)$ and $\mathcal{N}_{4}=(I-\psi(A)) B(Y)(I-\psi(A))$. As $\mathcal{M}_{1}=\mathbb{C} A$ we have $\psi\left(\mathcal{M}_{1}\right)=\mathbb{C} \psi(A) \subseteq \mathcal{N}_{1}$. Take $S \in \mathcal{M}_{2}$. Then $A+\alpha S$, as well as $\psi(A)+\alpha \psi(S)$, is idempotent for any $\alpha \in \mathbb{C}$. Hence we have

$$
\psi(A) \psi(S)+\psi(S) \psi(A)+\alpha \psi(S)^{2}=\psi(S)
$$

and this yields $\psi(S)^{2}=0$ and $\psi(S)=(I-\psi(A)) \psi(S) \psi(A)+\psi(A) \psi(S)(I-\psi(A)) \in$ $\mathcal{N}_{2} \oplus \mathcal{N}_{3}$. Therefore, $\psi\left(\mathcal{M}_{2}\right) \subseteq \mathcal{N}_{2} \oplus \mathcal{N}_{3}$, and similarly, $\psi\left(\mathcal{M}_{3}\right) \subseteq \mathcal{N}_{2} \oplus \mathcal{N}_{3}$. Let 
$S \in \mathcal{M}_{4}$ be any idempotent operator; then $A S=S A=0$ and so $\psi(A) \psi(S)=$ $\psi(S) \psi(A)=0$. It follows that $\psi(S) \in \mathcal{N}_{4}$. Note that $\mathcal{M}_{4}=\left\{\left[\begin{array}{ll}0 & 0 \\ 0 & C\end{array}\right]: C \in\right.$ $B(\operatorname{ker} A)\}$ with respect to the space decomposition $X=\operatorname{ran} A \oplus \operatorname{ker} A$. If $P=$ $\left[\begin{array}{ll}P_{11} & P_{12} \\ P_{21} & P_{22}\end{array}\right]$ is an idempotent operator in $B(X)$, then $P_{22}-P_{22}^{2}=P_{21} P_{12}$ is a rank one operator and therefore $P_{22}$ is the sum of an idempotent operator and a rank one operator. This obviously yields that every $C \in B(\operatorname{ker} A)$ is a linear combination of idempotents in $B(\operatorname{ker} A)$. Thus we have $\psi\left(\mathcal{M}_{4}\right) \subseteq \mathcal{N}_{4}$. Therefore, we get $\psi(B(X)) \subseteq \mathbb{C} \psi(A) \oplus \mathcal{N}_{2} \oplus \mathcal{N}_{3} \oplus \mathcal{N}_{4}$. However, $\psi$ is onto, so we must have $\mathbb{C} \psi(A)=\mathcal{N}_{1}=\psi(A) B(Y) \psi(A)$. This implies that $\psi(A)$ is rank one or zero.

Now, by [3 Thm. 3.2], $\left.\psi\right|_{\mathcal{F}(X)}$ is a Jordan homomorphism. Since $\mathcal{F}(X)$ is locally a matrix algebra, $\left.\psi\right|_{\mathcal{F}(X)}=\xi+\eta$, where $\xi: \mathcal{F}(X) \rightarrow B(Y)$ is a homomorphism and $\eta: \mathcal{F}(X) \rightarrow B(Y)$ is an anti-homomorphism. Since $\left.\psi\right|_{\mathcal{F}(X)} \neq 0$, there is a rank one idempotent $A$ such that $\psi(A) \neq 0$. Then, as shown above, $\psi(A)=\xi(A)+\eta(A)$ is a rank one idempotent as well as the sum of idempotents. It follows that either $\xi(A)=0$ or $\eta(A)=0$. Thus, at least one of $\xi$ and $\eta$ has a nonzero kernel. Since the kernels of homomorphisms and anti-homomorphisms are ideals and since the only nonzero ideal of $\mathcal{F}(X)$ is $\mathcal{F}(X)$ itself, we get either $\left.\psi\right|_{\mathcal{F}(X)}=\xi$ or $\left.\psi\right|_{\mathcal{F}(X)}=\eta$.

Assume $\left.\psi\right|_{\mathcal{F}(X)}=\xi$. It is clear that $\xi$ is injective, and consequently rankpreserving. By [7] we know that there exist injective operators $T \in B(X, Y)$ and $R \in B\left(X^{*}, Y^{*}\right)$ such that $\psi(x \otimes f)=T x \otimes R f$ for every $x \in X$ and every $f \in X^{*}$, where $x \otimes f$ stands for the rank one operator $u \mapsto\langle u, f\rangle x$. Since $\psi$ is homomorphic on $\mathcal{F}(X)$, for any $F \in \mathcal{F}(X)$ we have

$$
\psi(F) T x \otimes R f=\psi(F x \otimes f)=T F x \otimes R f .
$$

So

$$
\psi(F) T=T F \quad \text { for every } F \in \mathcal{F}(X) .
$$

Next, taking any idempotent operator $P$ in $B(X)$, let us prove $S=T P-\psi(P) T=$ 0 . It suffices to show that $S Q=0$ for every rank one idempotent operator $Q$ satisfying $P Q=Q P=0$ or $P Q=Q P=Q$. If $P Q=Q P=0$, then $\psi(P) \psi(Q)=0$ and hence $S Q=-\psi(P) T Q=-\psi(P) \psi(Q) T=0$. If $P Q=Q P=Q$, then $\psi(P) \psi(Q)=\psi(Q)$ and again we get $S Q=T Q-\psi(P) T Q=T Q-\psi(Q) T=0$. Therefore, we have proved that

$$
\psi(A) T=T A
$$

for every idempotent operator, and consequently for every operator $A$ in $B(X)$, since $X$ is of infinite multiplicity. It is now obvious that $T$ is invertible, as the range of $T$ is invariant under $\psi(B(X))=B(Y)$, which implies the surjectivity of $T$. Thus we have

$$
\psi(A)=T A T^{-1} \quad \text { for every } A \in B(X) .
$$

If $\left.\psi\right|_{\mathcal{F}(X)}=\eta$ is an anti-homomorphism, we have $\psi(x \otimes f)=W f \otimes R x$ and $\psi(F)^{*} R=R F$ for $F \in \mathcal{F}(X)$, where $W \in B\left(X^{*}, Y\right)$ and $R \in B\left(X, Y^{*}\right)$ are injective. A similar argument shows that $\psi(A)^{*} R=R A$ for all $A$ in $B(X)$, and hence $R$ is invertible. It follows that $\psi$ is an anti-homomorphism from $B(X)$ onto $B(Y)$. 
It is proved in [2] that a bijective idempotent-preserving linear map from $B(H)$ onto itself is either an automorphism or an anti-automorphism, where $H$ is a complex Hilbert space. It is also proved in [15] that a surjective linear map $\phi$ on $B(H)$ preserves $r$-potents in both directions (i.e., $\phi(A)^{r}=\phi(A) \Leftrightarrow A^{r}=A$ ) if and only if either $\phi$ is an automorphism multiplied by an $(r-1)$-th root of 1 , or an anti-automorphism multiplied by an $(r-1)$-th root of 1 . Our following corollary generalizes these results.

Corollary 3.7. Let $H$ and $K$ be complex Hilbert spaces and $f$ a polynomial in $\mathcal{P}_{0}$. Let $\phi: B(H) \rightarrow B(K)$ be a linear map which is surjective and is nonzero on $\mathcal{F}(X)$. Then $\phi$ preserves elements annihilated by $f(x)$ if and only if there exists a scalar $\lambda$ which is a root of 1 satisfying $\lambda \mathcal{Z}(f)=\mathcal{Z}(f)$ and there exists an invertible operator $T \in B(H, K)$ such that either $\phi(A)=\lambda T A T^{-1}$ for every $A$, or $\phi(A)=\lambda T A^{t} T^{-1}$ for every $A$, where $A^{t}$ is the transpose of $A$ relative to a basis.

Finally we give a characterization of homomorphisms between Banach algebras in terms of linear preservers.

Theorem 3.8. Let $\mathcal{A}$ and $\mathcal{B}$ be complex unital algebras and $\phi: \mathcal{A} \rightarrow \mathcal{B}$ a linear map. Let $f$ be a polynomial of degree greater than 1 and with no repeated roots such that $f(0)=0$. If $\mathcal{A}$ is a Banach algebra, then the following statements are equivalent.

(1) $\phi_{2}$ preserves elements annihilated by $f$.

(2) $\phi$ is a homomorphism multiplied by an $r$-potent element with spectrum contained in $\{\lambda: \lambda \mathcal{Z}(f)=\mathcal{Z}(f)\}$.

Proof. $(2) \Longrightarrow(1)$ is obvious.

$(1) \Longrightarrow(2)$. Let $B=\phi(I)$. By Corollary 2.4, there exists a Jordan homomorphism $\psi$ such that $\phi=B \psi$ and $\psi(C) \psi\left(C^{-1} A C\right)=\psi(A) \psi(C)$ for all $A$ and all invertible $C$ in $\mathcal{A}$. As in the proof of Corollary 2.5, without loss of generality, we may assume that $\psi$ is unital. Then $\psi$ preserves invertibility and $\psi\left(C^{-1}\right)=\psi(C)^{-1}$. Note that, since $\psi$ is a Jordan homomorphism, we also have $\psi(E A E)=\psi(E) \psi(A) \psi(E)$ for any $A$ and $E$ in $\mathcal{A}$. Thus, if $C$ is invertible, we have

$$
\psi\left(C^{2} A\right)=\psi\left(C(C A C) C^{-1}\right)=\psi(C)^{2} \psi(A) .
$$

Now since $\mathcal{A}$ is a Banach algebra, the spectrum of every element in $\mathcal{A}$ is a nonempty compact subset of $\mathbb{C}$. Take a nonzero $\lambda \in \mathbb{C} \backslash\left(\operatorname{Sp}(C) \cup \operatorname{Sp}\left(C^{2}\right)\right)$, where $\operatorname{Sp}(T)$ denotes the spectrum of $T$ in $\mathcal{A}$. Replacing $C$ by $\lambda-C$ in (3.1), we get

$$
\psi(C A)=\psi(C) \psi(A)
$$

for all $A$ and invertible $C$. Now for any $A$ and $W$ in $\mathcal{A}$, take $\lambda \in \mathbb{C}$ so that $\lambda-W$ is invertible. Then by $(3.2) \psi((\lambda-W) A)=(\lambda-\psi(W)) \psi(A)$, which again implies that $\psi(W A)=\psi(W) \psi(A)$. Hence $\psi$ is homomorphic.

Remark 3.2. From the proof of the above theorem it is clear that if $\mathcal{A}$ and $\mathcal{B}$ are any unital algebras over $\mathbb{C}$ and if $\mathcal{A}$ has the property that $\operatorname{Sp}(a) \neq \mathbb{C}$ and $\operatorname{Sp}(c) \cup \operatorname{Sp}\left(c^{2}\right) \neq \mathbb{C} \backslash\{0\}$ whenever $c$ is invertible, then the conclusion of Theorem 3.9 remains true.

\section{ACKNOWLEDGMENTS}

Most of this work was done during the first author's visit at the Department of Mathematics, University of New Hampshire, from September 1999 to March 2000. 
He gives thanks to Liming Ge, and especially to Don Hadwin, who contributed many ideas and suggestions to the present paper.

\section{REFERENCES}

[1] M. Bresar and P. Semrl, Linear maps preserving the spectral radius. J. Funct. Anal, 142 (1996), 360-368. MR 97i:47070

[2] M. Bresar and P. Semrl, On local automorphisms and mappings that preserve idempotents, Studia Math. 113 (3) (1995), 101-108. MR 96i:47058

[3] M. Bresar and P. Semrl, Mappings which preserve idempotents, local automorphisms, and local derivation. Can. J. Math. 45(3), (1993), 483-496. MR 94k:47054

[4] C. H. Chan and M. H. Lim, Linear preserves on powers of matrices, Linear Algebra Appl. 162-164 (1992), 615-626. MR 92k:15009

[5] G. Frobenius, Uber die Darstellung der endlichen Gruppen durch lineare Substitutionen, Sitzungsber. Deutsch. Akad. Wiss. Berlin (1897), 994-1015.

[6] Hadwin Lunch Bunch, Local multiplications on algebras spanned by idempotents, Linear and Multilinear Algebra, 37 (1994), 259-263. MR 96f:47070

[7] J. Hou, Rank preserving linear maps on $B(X)$, Sci. in China, 32A (1989), 929-940. MR 92b:47052

[8] R. Howard, Linear maps that preserve matrices annihilated by a polynomial, Linear Algebra Appl. 30 (1980), 167-176. MR 81h:15002

[9] A. A. Jafarian and A. R. Sourour, Spectrum preserving linear maps, J. Funct. Anal, 66 (1989), 255-261. MR 87m:47011

[10] S. Kantor, Theorie der Aquivalenz von linearen $\infty$ Scharen bilinearer Formen, Sitzungsber. Munchener Akad., (1897), 367-381.

[11] C-K. Li and N-K. Tsing, Linear preserver problems: A brief introduction and some special techniques, Lin. Alg. Appl. 162- 164 (1992), 217-235. MR 93b:15003

[12] M. Marcus, All linear operators leaving the unitary group invariant, Duke Math. J. 26: 155163 (1959). MR 21:54

[13] M. Marcus, Linear operations on matrices, Amer. Math. Monthly, 69: 837-847 (1962). MR 26:5007

[14] M. Omladic, On operators preserving commutativity, J. Funct. Anal, 66 (1986), 105-122. MR $87 \mathrm{k}: 47080$

[15] M. Omladic and P. Semrl, Linear mappings that preserve potent operators, Proc. Amer. Math. Soc., 123(4) (1995), 1069-1074. MR 95e:47049

[16] P. Semrl, Linear mappings that preserve operators annihilated by a polynomial, J. Oper. Theo. 36 (1996), 45-58. MR 97i:47071

Department of Mathematics, Shanxi Teachers University, Linfen 041004, People's Republic of China

E-mail address: jhou@dns.sxtu.edu.cn

Institute of Mathematics, Fudan University, Shanghai, 200433, People's Republic of CHINA

E-mail address: 970004@fudan.edu.cn 